\title{
Age: HIV knows no boundary
}

Olivia C Smibert

MBBS, DipTropMed

Medical Registrar $^{1}$

Michelle Ananda-Rajah MBBS, FRACP, PhD

Infectious Diseases and

General Medicine Physician ${ }^{1}$

Jessica O'Brien

Medical Registrar

\section{Kumar Visvanathan}

MBBS, PhD

Clinical Director Medicine and Emergency Services and Infectious Diseases Physician²

1 Alfred Hospital, Melbourne, VIC.

2 St Vincent's Hospital, Melbourne, VIC.

O.Smibert@ alfred.org.au

\section{Clinical record}

A 66-year-old retired man was referred to a regional hospital for investigation of a prolonged illness characterised by a 9-month history of profound lethargy, loss of appetite, episodic explosive watery diarrhoea without blood or mucus, and weight loss of $45 \mathrm{~kg}$, resulting in a body mass index of $19 \mathrm{~kg} / \mathrm{m}^{2}$. His functional status had deteriorated such that he was mostly bed-bound and required a walking stick to mobilise. The patient's syndrome had been extensively investigated in the outpatient setting by other clinicians in the region without any causative abnormality identified.

The patient described intermittent fevers and sweats and reported a propensity to pick up "every common infection going around". He had a background of two coronary angioplasties, mild depression, hypertension and dyslipidaemia, for which he was receiving appropriate treatment. His regular medications included citalopram, aspirin, simvastatin and metoprolol, and he had no history of medication allergies. He was married and had adult children from a previous marriage. He denied a history of smoking or use of recreational drugs, and reported drinking alcohol on social occasions.

On physical examination, he was cachectic and afebrile, with normal vital signs and mildly dry mucous membranes. Results of a clinical examination were otherwise unremarkable, with no organomegaly, lymphadenopathy or rashes evident.

The patient had been extensively investigated over the preceding 9 months by three specialist clinicians. These investigations included upper and lower gastrointestinal endoscopies, which were unremarkable, and chest and abdominal computed tomography scans, which showed only mild non-specific intra-abdominal lymphadenopathy. Blood tests had shown a mild normocytic anaemia (haemoglobin level, 121 g/L [reference interval (RI), 128$175 \mathrm{~g} / \mathrm{L}]$ ), ferritin level of $2400 \mu \mathrm{g} / \mathrm{L}$ (RI, 30-600 $\mathrm{g} / \mathrm{L}$ ), erythrocyte sedimentation rate of $53 \mathrm{~mm} / \mathrm{h}(\mathrm{RI}, 1-10 \mathrm{~mm} / \mathrm{h})$, C-reactive protein level of $4.5 \mathrm{mg} / \mathrm{L}$ (RI, $0-5 \mathrm{mg} / \mathrm{L}$ ), normal white blood cell count and no evidence of lymphopenia. Serum electrolyte levels and results of liver function and renal function tests were also within reference intervals. Serological test results confirmed past exposure to cytomegalovirus, Epstein-Barr virus and toxoplasmosis and were negative for hepatitis $A, B$ and $C$. Levels of tumour markers including carcinoembryonic antigen, CA-125, CA19-9 and prostate-specific antigen were within reference intervals, as were serum cortisol and plasma copper levels. Three stool specimens collected on consecutive days tested negative for white blood cells, red blood cells, pathogenic cysts, ova and parasites.

Given the clinical syndrome, occult malignancy was considered and peripheral lymphocyte flow cytometric analysis was performed, which showed a total CD4+ count of 66 cells/ $/ \mathrm{L}$ (RI, 4101545 cells $/ \mu \mathrm{L}$ ). The patient denied risk factors for HIV acquisition and reported having a negative HIV test result 10 years earlier. He denied any intravenous drug use or unprotected sexual intercourse outside his marriage. On subsequent questioning, he did not report an illness consistent with seroconversion illness.

HIV testing was conducted after counselling and consent and gave a positive result, with a viral load of $>1000000$ copies $/ \mathrm{mL}$. The patient was subsequently screened for opportunistic infections, including tuberculosis and sexually transmitted infections. HIV genotyping identified no resistance mutations and he commenced daily treatment with a combined tablet (Atripla; Gilead) containing efavirenz $600 \mathrm{mg}$, emtricitabine $200 \mathrm{mg}$ and tenofovir $300 \mathrm{mg}$. He was discharged home after 3 weeks without emergence of immune reconstitution inflammatory syndrome. On follow-up 6 weeks later the patient had gained $6 \mathrm{~kg}$ in weight, diarrhoea had ceased and he was able to ambulate independently without gait aids over $100 \mathrm{~m}$. His partner subsequently tested negative to HIV.
D espite better awareness of HIV, late diagnoses - defined as a CD4+ count of $<350$ cells $/ \mu \mathrm{L}$ on testing, or presentation with an AIDS-defining illness - continue to occur, even in wealthy countries. The rate of new HIV diagnosis in Australia has begun to rise in recent years, and older people are increasingly represented. ${ }^{1-3}$ Multiple studies have demonstrated that a diagnosis of HIV over the age of 50 years is associated with more advanced disease at the time of diagnosis, and that age is an independent predictor of clinical progression to AIDS or death. 2,3 Therefore, while older people with HIV are few in absolute numbers, they experience significant HIV-associated morbidity and mortality.

The clinical manifestations of HIV include an acute seroconversion illness, followed by a period of latency typically lasting 5-10 years, with later protean clinical manifestations occurring with declining immunological function. Patients often have multiple clinical encounters before the diagnosis is made, emphasising the importance of opportunistic testing even when risk factors are not reported.
Factors that are likely to contribute to low rates of testing and late or missed diagnosis of HIV include older age, poor awareness and misconceptions regarding HIV risk factors. ${ }^{3-5}$ In addition, older people are less likely to be screened for HIV. ${ }^{4}$ One study found that adults aged 65 years or older had the lowest HIV testing rate $(11.4 \%)$ compared with other age groups, with the highest rate seen among adults aged $25-34$ years $(53.5 \%) .{ }^{5}$ Health care providers consistently under-recognise the possibility of HIV in older patients because specific HIV or opportunistic infection symptoms can mimic other diseases frequently associated with older age. ${ }^{3}$ The patient reported here presented with a syndrome compatible with stage IV HIV and was diagnosed late despite numerous clinical encounters; HIV testing was only initiated after a low CD4+ cell count was indirectly identified on peripheral lymphocyte flow cytometry as part of an investigation into suspected malignancy.

The Australian HIV epidemic is largely dominated by diagnoses associated with male-to-male sexual transmission in young men, with over $63 \%$ of all new diagnoses 


\section{Lessons from practice}

- New diagnoses of HIV are continuing to rise in the Australian population, and older people are increasingly represented.

- In older people, a diagnosis of HIV can be missed or delayed if clinicians do not consider the diagnosis.

- Clinicians should consider HIV testing in any patient presenting with an HIV-compatible clinical syndrome, even when traditional risk factors are not reported.

- Clinicians outside specialised clinics should have a low threshold for offering HIV testing, given the protean manifestations of the disease, especially in its advanced stage.

made in this cohort. ${ }^{1,6,7}$ The median age of Australian men diagnosed with HIV in 2011 was 37 years. ${ }^{1}$ Geographically, young to middle-aged male patients tend to be clustered in urban areas, with most diagnoses ( $>88 \%$ ) made in metropolitan centres. ${ }^{8,9}$ As these patients are subsequently treated in a few specialised clinics, rural and general practitioners are relatively underexposed to patients with HIV. It is important that members of the medical community outside specialised clinics remain vigilant for HIV in their patients.

This case highlights the importance of early consideration of HIV infection in all patients presenting with symptoms compatible with a diagnosis of HIV, not only those with traditionally recognised risk factors for infection. Given that the number of HIV infections in Australia is rising annually, and there are serious public health implications associated with missed or delayed diagnosis, it is important to heighten awareness of HIV across all medical specialties, including in rural and remote centres. In Australia, HIV testing is accessible and effective treatment available, and they should be readily offered to avoid the devastating consequences of advanced infection.

Competing interests: No relevant disclosures.

1 The Kirby Institute. HIV, viral hepatitis and sexually transmissible infections in Australia. Annual surveillance report 2012. Sydney: The Kirby Institute, University of New South Wales, 2012. http://kirby.unsw.edu.au/sites/ default/files/hiv/resources/2012AnnualSurvReport.pdf (accessed Aug 2014).

2 Iwuji CC, Churchill D, Gilleece Y, et al. Older HIV-infected individuals present late and have a higher mortality: Brighton, UK cohort study. BMC Public Health 2013; 13: 397

3 Nguyen N, Holodniy M. HIV infection in the elderly. Clin Interv Aging 2008; 3: 453-472.

4 Lekas HM, Schrimshaw EW, Siegel K. Pathways to HIV testing among adults aged fifty and older with HIV/AIDS. AIDS Care 2005; 17: 674-687.

5 US Centers for Disease Control and Prevention. HIV/AIDS surveillance report. Cases of HIV infection and AIDS in the United States and dependent areas, 2005. Vol. 17. Atlanta: US Department of Health and Human Services, CDC, 2007. http://www.cdc.gov/hiv/pdf/statistics_2005_HIV_Surveillance_ Report_vol_17.pdf (accessed Aug 2014).

6 Guy RJ, McDonald AM, Bartlett MJ, et al. Characteristics of HIV diagnosis in Australia 1993-2006. Sex Health 2008; 5: 91-96.

7 Murray JM, McDonald AM, Law MG. Rapidly ageing HIV epidemic among men who have sex with men in Australia. Sex Health 2009; 6: 83-86.

8 Jansson J, Wilson D, Watson J. Mapping HIV outcomes: geographical and clinical forecasts of numbers of people living with HIV in Australia. Sydney: National Association of People Living with HIV/AIDS and the National Centre in HIV Epidemiology and Clinical Research, University of New South Wales, 2010. http://kirby.unsw.edu.au/sites/default/files/hiv/attachment/ FinalModHIVoutcomesAusRep.pdf (accessed Aug 2014).

9 Victorian Government Department of Health. HIV/AIDS monthly surveillance report. June 2013. Melbourne: Department of Health, 2013. 